\title{
The Endcap Disc DIRC for PANDA at FAIR
}

M. $\operatorname{Schmidt}\left({ }^{1}\right)\left({ }^{*}\right)$, A. Ali $\left({ }^{2}\right)\left({ }^{3}\right)$, A. Belias $\left({ }^{2}\right)$, R. Dzhygadlo $\left({ }^{2}\right)$, A. $\operatorname{Gerhardt}\left({ }^{2}\right)$, M. Krebs $\left({ }^{2}\right)\left({ }^{3}\right)$, D. Lehmann $\left({ }^{2}\right)$, K. Peters $\left({ }^{2}\right)\left({ }^{3}\right)$, G. $\operatorname{Schepers}\left({ }^{2}\right)$, C. Schwarz $\left({ }^{2}\right)$, J. Schwiening $\left({ }^{2}\right)$, M. Traxler $\left({ }^{2}\right)$, L. $\operatorname{Schmitt}\left({ }^{4}\right)$, M. Böhm $\left({ }^{5}\right)$, A. Lehmann $\left({ }^{5}\right)$, M. Pfaffinger $\left({ }^{5}\right)$, S. Stelter $\left({ }^{5}\right)$, F. Uhlig $\left({ }^{5}\right), \operatorname{L} . \operatorname{Brück}\left({ }^{1}\right), \operatorname{M} . \operatorname{Düren}\left({ }^{1}\right)$, E. Etzelmüller $\left({ }^{1}\right)$, K. Föhl $\left({ }^{1}\right)$, A. Hayrapetyan $\left({ }^{1}\right), \operatorname{K} . \operatorname{Kreutzfeldt}\left({ }^{1}\right)$, J. Rieke $\left({ }^{1}\right)$, T. Wasem $\left({ }^{1}\right)$, P. Achenbach $\left({ }^{6}\right)$, M. Cardinali $\left({ }^{6}\right)$, M. Hoek $\left({ }^{6}\right)$, W. Lauth $\left({ }^{6}\right)$, S. Schlimme $\left({ }^{6}\right)$, C. Sfienti $\left({ }^{6}\right)$ and M. Thiel $\left({ }^{6}\right)$ on behalf of the PANDA COLLABORATION

$\left({ }^{1}\right)$ II. Physikalisches Institut, Justus Liebig-University of Giessen - Giessen, Germany

$\left.{ }^{2}\right)$ GSI Helmholtzzentrum für Schwerionenforschung GmbH - Darmstadt, Germany

$\left({ }^{3}\right)$ Goethe University - Frankfurt a.M., Germany

(4) FAIR, Facility for Antiproton and Ion Research in Europe - Darmstadt, Germany

$\left({ }^{5}\right)$ Friedrich-Alexander University of Erlangen-Nuremberg - Erlangen, Germany

$\left.{ }^{6}\right)$ Institut für Kernphysik, Johannes-Gutenberg-University of Mainz - Mainz, Germany

received 5 February 2019

Summary. - The Endcap Disc DIRC for the PANDA experiment is going to provide an excellent particle identification up to particle momenta of $4 \mathrm{GeV} / \mathrm{c}$. It covers the polar angles from $5^{\circ}$ to $22^{\circ}$ and guarantees a separation power of more than 3 standard deviations (s.d.) for pions and kaons in the required phase space. The simulated detector performance has been validated during several testbeam campaigns. Additionally, the transmission losses of the optical filter has been investigated to estimate the filter performance at the end of the PANDA lifetime.

\section{1. - The Endcap Disc DIRC in PANDA}

The Antiproton Annihilation at Darmstadt (PANDA) experiment is designed as a fixed target experiment for $\bar{p} p$ collisions up to an antiproton beam momentum of $15 \mathrm{GeV} / \mathrm{c}$ and a maximum interaction rate of $20 \mathrm{MHz}$ in the High-Energy Storage Ring (HESR) that is a part of the future Facility for Antiproton and Ion Research (FAIR). The goal of PANDA is to cover a large variety of physics studies in the strange and charm sectors [1].

$\left.{ }^{*}\right)$ Corresponding author. E-Mail: mustafa.a.schmidt@exp2.physik.uni-giessen.de 


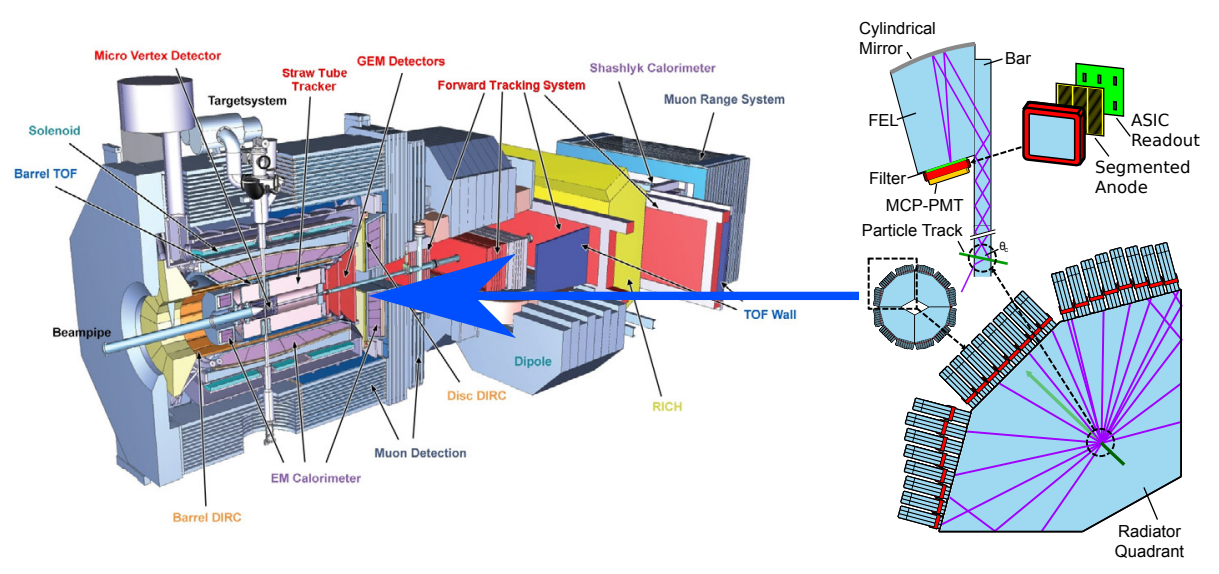

Fig. 1. - The PANDA experiment (left) and a sketch of different components of the Endcap Disc DIRC in PANDA (right).

PANDA needs an excellent Particle Identification (PID) which will be achieved with two Cherenkov detectors in the target spectrometer: the Endcap Disc DIRC (EDD) and the Barrel DIRC. Additionally, a third Cherenkov detector for very small polar angles below $5^{\circ}$ will be placed in the forward spectrometer.

The EDD for PANDA is a new type of Cherenkov detector that is based on photon propagation via internal reflection in a radiator plate [2]. The detector consists of 4 independent quadrants made of fused silica with highly polished surfaces in order to preserve the photon angles while propagating through the radiator and to guarantee a minimum amount of photon losses.

As shown in fig. 1, the detector is placed at the forward endcap of the PANDA target spectrometer and is designed to separate charged pions and kaons up to a momentum of $4 \mathrm{GeV} / \mathrm{c}$ in the polar angle range between $5^{\circ}$ and $22^{\circ}$ with a separation power of more than 3 s.d. Larger polar angles between $22^{\circ}$ and $140^{\circ}$ will be covered by the Barrel DIRC [3]. Both detectors together cover almost the full phase space of the produced kaons.

Once the Cherenkov light is produced along the particle track, each photon propagates to the outer rim. There it enters one of the 72 Focusing Elements (FELs) that are glued to each radiator quadrant and contain a cylindrical mirror to focus the incoming photons (fig. 1). Three consecutive FELs are combined to one Readout Module (ROM) with an attached Micro Channel Plate Photo Multiplier Tube (MCP-PMT). Optical filters are used to minimize a deterioration of the resolution as a result of chromatic errors.

The anode of the MCP-PMT is segmented into small pixels to receive the information about the exact photon position. The pixel number, a time stamp, and the Time over Threshold (ToT) value will be acquired by the TOFPET ASIC that has been designed by the company PETsys Electronics S.A. The intrinsic time resolution of the readout is $50 \mathrm{ps}$ and therefore suitable for the acquisition of fast MCP-PMT signals [4].

The cylindrical shape of the mirror ensures a linear dependency between the pixel number and photon angle that is used for either the direct computation of the Cherenkov angle $\theta_{C}$ or the calculation of a theoretical hit pattern. This algorithm is based on likelihood values by taking the tracking information of the charged particle into account [5]. 

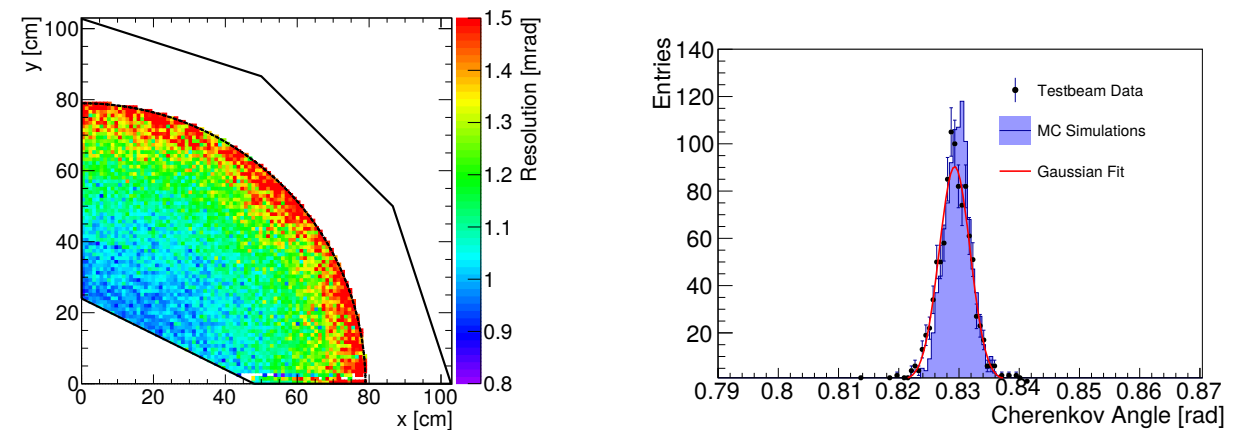

Fig. 2. - The simulated detector resolution for one quadrant of the final detector (left) and the measured resolution from the DESY testbeam campaign done in 2016 by using event combination with an inset sketch of the measurement setup (right).

\section{2. - Performance studies of the EDD}

The left side of fig. 2 illustrates the simulated detector resolution for $\pi^{+}$and $K^{+}$with a momentum of $4 \mathrm{GeV} / \mathrm{c}$ as a function of the particle position on one radiator quadrant. The simulations have been performed with the full detector geometry implemented in the PandaRoot framework [6]. It turns out that the desired resolution of 1.4 mrad can be achieved almost everwhere on the radiator surface with the proposed design.

On the right side of fig. 2 the analysis results from a testbeam campaign in 2016 at DESY are presented. Several scans regarding the angle of incidence and horizontal respectively vertical scans have been performed [7].

The overall detector resolution of $2.5 \mathrm{mrad}$ has been obtained with a combination of events from the vertical position scan in order to simulate the existance of 30 independent FELs while only one physical FEL has been taken into account. The matching of the two histograms show that the measured data fit very well to the predicted resolution that are taken from dedicated Monte-Carlo simulations.

The reason for not being able to obtain a resolution of better than $2 \mathrm{mrad}$ can be explained by the absence of optical filters in the DESY setup and the larger background due to the use of an electron beam with a momentum of $3 \mathrm{GeV} / \mathrm{c}$. The effect of an increased angular straggling and energy loss of the electron beam together with larger beam size uncertainties set an upper limit for the maximum achievable detector performance.

A new testbeam campaign with an updated setup and a new TOFPET-ASIC version has been completed in August 2018 and the data are currently being analyzed. The goal is to show a clear separation between two particle species in order to proof the working principle of the EDD prototype regarding photon yield and spatio-temporal resolution with the actual design parameters.

\section{3. - Estimated radiation damage}

The simulations for obtaining the estimated radiation dose in the PANDA experiment have been performed with the Dual Parton Model (DPM) generator that is implemented in the PandaRoot framework including all PANDA subdetectors. This particle generation 

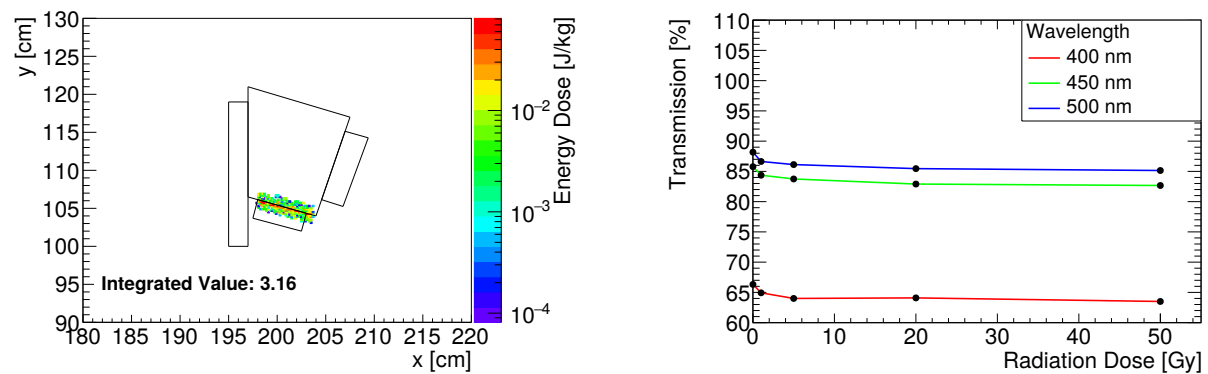

Fig. 3. - The simulated radiation dose for one optical filter (left) in the final setup and the measured transmission behavior as a function of the radiation dose for a sample filter (right).

algorithm has been originally designed with the intention to study background rates of charged particles and resulting detector occupancies. For the particle generation process based on the anti-proton beam momentum, a large- $\mathrm{N}$ expansion of non-perturbative Quantum Chromodynamics (QCD) and the Reggeon field theory is used to compute the kinematics of the created hadrons [8].

The optical filter between the ROM and the MCP-PMT can be seen as the critical component regarding radiation damage in the EDD. The left side of fig. 3 shows the simulated radiation dose for one filter in the final detector setup after a PANDA lifetime of 10 years with a duty cycle of $50 \%$. The results indicate an overall radiation dose of 3.16 Gy for each filter volume.

The right side of fig. $\mathbf{3}$ represents the measured transmission of a longpass color filter FGL400S from the company Thorlabs with cut-on wavelength of $400 \mathrm{~nm}$ as a sample measurement [9]. The transmission loss for radiation damages of around 3 Gy could be estimated to $1.5 \%$ at $500 \mathrm{~nm}$ and $3.5 \%$ at $400 \mathrm{~nm}$. In case of a 10 times larger radiation dose, the transmission loss has been determined as values below $4.5 \%$ depending on the wavelength of the transmitted light. According to the latest simulation predictions, this high radiation dose will not be attained during the whole lifetime of PANDA.

\section{REFERENCES}

[1] Iazzi F., AIP Conf. Proc., 1743 (2016) 050006.

[2] Düren M. et al., Nucl. instrum Methods Phys. Res. Sect. A, 876 (198) 2017.

[3] Dzhygadlo R. et al., The Barrel DIRC Detector for the PANDA Experiment at FAIR, in Proceedings of International Conference on Technology and Instrumentation in Particle Physics 2017. TIPP 2017, edited by Liu Z.A., Springer Proceedings in Physics, vol. 212 (Springer, Singapore) 2018, pp. 123126.

[4] Rolo M. D. et al., J. Instrum., 8 (2013) C02050.

[5] Schmidt M. et al., J. Instrum., 12 (2017) C12051.

[6] Spataro S., J. Phys.: Conf. Ser., 331 (2011) 032031.

[7] Fohl K. et al., J. Instrum., 13 (2018) C02002.

[8] Capella A., Introduction to the Dual Parton Model (Springer US, Boston, MA) 1994, pp. 287-310.

[9] Bruck L., Measurement and qualication of optical filters and adhesives for the PANDA Endcap Disc DIRC, Bachelor's Thesis (2018). 